\title{
Experimental Evaluation of IEEE 802.15.4/ZigBee for Multi-Patient ECG Monitoring
}

\author{
Helena Fernández-López ${ }^{1}$, José H. Correia ${ }^{1}$, Ricardo Simões ${ }^{2,3}$ and José A. Afonso ${ }^{1}$ \\ ${ }^{1}$ Industrial Electronics Engineering Department, University of Minho, Guimarães, Portugal \\ ${ }^{2}$ Institute of Polymers and Composites, University of Minho, Guimarães, Portugal \\ ${ }^{3}$ Polytechnic Institute of Cávado and Ave, Barcelos, Portugal \\ ${ }^{1}\{$ hlopez, jose.afonso, higino.correia\}@dei.uminho.pt \\ ${ }^{2}$ rsimoes@dep.uminho.pt
}

\begin{abstract}
IEEE 802.15.4/ZigBee wireless sensor networks (WSNs) are a promising alternative to cabled systems for patient monitoring in hospitals. Some areas where monitoring systems based on WSNs can be successfuly used are ambulatory, waiting and triage rooms, post-op, and emergency rooms. The low power and small size ZigBee devices have the ability to form selfconfiguring networks that can extend themselves through a hospital wing or floor. Using spatially distributed networks, it is possible to cover an extended area and serve several patients. However, the low data rate protocols provided by IEEE 802.15 .4 poses several challenges, mainly because its protocols were primarily designed to operate in low traffic load scenarios but some vital signs sensors generate a large volume of data. This work presents an experimental evaluation of the performance of multi-hop ZigBee networks comprised of several nodes that carry the traffic of wearable electrocardiogram (ECG) sensors. The results indicate that star networks can relay $100 \%$ of the traffic generated by at least 12 ECG nodes. In tree topologies, the increase of the network traffic load reduces the performance but even these networks can reliably relay the traffic of a considerable number of ECG nodes.
\end{abstract}

Keywords: ZigBee, wireless sensor networks, e-Health, remote vital signs monitoring.

\section{Introduction}

Recently, the ZigBee Alliance released the ZigBee Health Care Profile Specification [1]. On top of this specification is the ISO/IEEE 11073 Personal Health Communication family of standards and the 104XX device specializations, which intends to enable plug-and-play interoperability between devices [2]. In the future ${ }_{2}$ it is expected that several manufacturers will introduce interoperable devices tailored to fulfill the needs of monitoring chronically-ill patients, independent living seniors, and in-patients being cared in assisted living facilities and in hospitals. Particularly in hospitals, several patients who presently are not being monitored could greatly benefit from continuous vital signs monitoring based on WSN technologies. The scenarios include emergency, waiting and triage rooms, and low-acuity patients in ambulatory, recovery wards, and post-operative care. 
WSNs are comprised of a large number of spatially distributed small devices with sensing, processing and radio communication capabilities [3]. Low power consumption, topology adaptation, and multi-hop routing, among other important features, make these networks attractive for pervasive healthcare applications. Despite of the evident benefits wireless communications can offer, some concerns prevent the spread use of WSNs for patient monitoring purposes. Depending on the sensors used to continuously monitor patients, large amounts of data have to be transmitted by the network, which is forced to operate in a high load scenario, where the network performance can be seriously affected.

In [4] we have estimated, based on simulations, the performance of ZigBee WSNs designed for continuous vital signs monitoring. These networks were evaluated in terms of scalability considering different topologies. This information was used to design the wireless vital sign monitoring system presented in [5], which is presently being tested on non-acute in-patients who have entered post-operating care. ECG, heart rate, and skin temperature sensors were designed to be minimally obtrusive and modular.

In this work, we present experimental measurements of the delivery ratio (DR) and end-to-end delay for networks consisting of ECG sensor nodes. Two sets of measurements were performed. The first one have employed development boards programmed to transmit raw ECG data sampled at $200 \mathrm{~Hz}$ while the second one have employed boards programmed to transmit ECG data compressed by a ratio of 2:1, what effectively reduced the number of packets to transmit by half. Star and tree topologies, with a crescent number of hops and sensors, were considered.

This paper is organized as follows. In the next section, we present the related work and their conclusions. In Section 3, we briefly review the IEEE 802.15.4 and ZigBee protocols. Section 4 presents the experimental results and, finally, in Section 5, the conclusions and future work are presented.

\section{Related Work}

Some previous works have discussed the performance of IEEE 802.15.4 based star networks used specifically for patient monitoring [6-8]; however, as far as we know, none have considered tree networks. Most works have also considered that each sensor node integrate a body area network (BAN) whose coordinator (usually, a PDA) also behaves as a gateway, relaying traffic to a wireless local area network. Although this approach has some advantages, as discussed in [9], we have observed that patient monitoring is evolving from a scenario where many sensors were used to monitor one patient, such as in [10], to a new one where only one or two devices can sense multiple vital signs and aggregate the traffic [11]. In this new scenario, the number of end devices (EDs) is reduced, what mitigates the need of BANs.

Other works have analyzed the performance of WSNs based on the IEEE 802.15.4 protocol, but few have presented experimental data. In [12] it is determined the maximum bandwidth efficiency and maximum packet delay for the $2.4 \mathrm{GHz}$ band considering a simple one sender and one receiver network. In [13], the authors have considered a non-beacon enabled star network consisting of ECG sensor nodes as a 
case study to analyze the network performance as a function of the payload size variation. They have observed that although the DR grows as the payload size increases, larger size packets may experience larger transmission delays. On the other hand, if the payload is small and frequent packet must be transmitted, the delay also tends to grow. In [14], simulation was used to evaluate the suitability of the IEEE 802.15.4 standard to a healthcare monitoring application, concluding that just three ECG sensor nodes can overload the network. But, these unfavorable results are due to the large bandwidth needed by the devices. Unlike the monitoring devices considered in these work, our system uses one-channel ECG sensors, which generate much less data but are still appropriate for monitoring purposes.

In [15], the authors have presented a fall detection system designed to monitor seniors living independently in a rural isolated village. The system infrastructure contains several routers distributed inside the houses and outdoors, covering different areas, depending on the transceiver power and antenna gain. The ZigBee coordinator is located on a local management center and is connected to a computer that works as a gateway relaying information to the Internet. As in most applications of WSNs (that usually consider low traffic loads), the successful implementation of this system does not guarantee the viability of using ZigBee networks for data-intensive scenarios, such as in the motivating application behind this work.

\section{IEEE 802.15.4 and ZigBee protocols}

The IEEE 802.15.4 standard [16] defines the physical (PHY) and medium access control (MAC) layers of the ZigBee network. The standard defines three operating frequency bands. The most used band ranges from $2400 \mathrm{MHz}$ to $2483.5 \mathrm{MHz}$ and is divided into sixteen channels that operate at a rate of $250 \mathrm{kbps}$. The MAC layer uses a contention based CSMA-CA scheme. An optional TDMA (Time Division Multiple Access) based scheme called guaranteed time slot (GTS) is also provided.

If the unslotted CSMA-CA is used, the device must wait for a random backoff interval before accessing the channel. After that, if the clear channel assessment (CCA) function indicates that the channel is idle, the device starts its transmission almost immediately. On the other hand, if the channel is busy, the device defers its transmission and calculates a new random backoff interval. The device can try to access the channel a maximum number of times, and when this limit is reached, the MAC layer discards the data and declares a channel access failure. Despite the mechanisms provided by the protocol, even when the channel can be accessed and messages get transmitted they might not reach the destination due to several factors, such as collisions, fading and interference.

The ZigBee protocol [17], developed by the ZigBee Alliance, stands on top of the IEEE 802.15.4 and defines the network and application layers. Star, tree and mesh topologies are supported. The network layer is responsible for routing frames to their intended destinations and provides functionalities such as network formation, address assignment and mechanisms to join and leave the network. It also provides an interface to the application layer, which holds application objects and provides mechanisms for discovering and binding devices. 


\section{Experimental Performance Analysis}

\subsection{Experimental results}

To measure the DR and the end-to-end delay, some test programs were specially developed. Two sets of tests were executed. In the first one, all ECG sensor nodes tried to send a packet with fifty 12-bit samples every $250 \mathrm{~ms}$, which corresponds to the raw data generated with a sampling rate of $200 \mathrm{~Hz}$. The second test considered that the data was compressed by a ratio of $2: 1$. In this case, all ECG end devices (ED) transmitted one packet every $500 \mathrm{~ms}$.

The experimental configuration is shown in Fig. 1. One of the Digital I/O (DIO) lines of the network coordinator (DIO2) was wire-connected to one of the DIO lines of a board which worked as the delay test ED (DIO10). Just before sending a packet, the application running in the delay test ED changes the DIO line state. This transition interrupts the coordinator, which reads and stores the counting value supplied by a 1 ms resolution clock. When the packet is received, the coordinator reads the counting value again and computes the interval between the two events, which results in a good approximation of the packet end-to-end delay. To determine the DR, we have programmed all EDs, including the delay test ED, to insert a sequential sequence number into the payload and checked for gaps in the sequence numbers of the received packets.

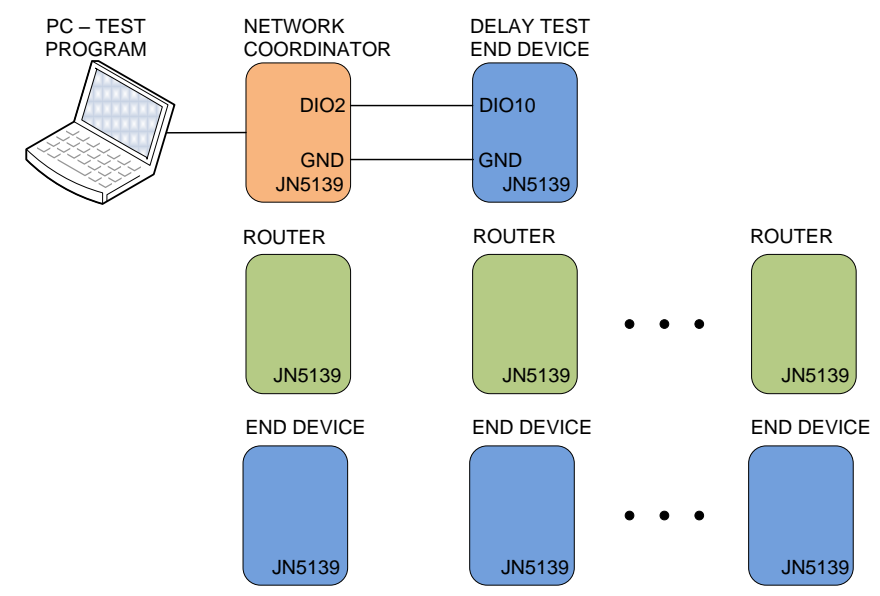

Fig. 1. Delivery ratio and delay test devices configuration.

All test boards used are based on the JN5139 module, from Jennic, and run the ZigBee 2004 stack [18]. To create tree networks from devices which are at the radio range of each other, it was necessary to prevent the ZigBee coordinator and routers from accepting new children during network formation. This behavior is accomplished by suppressing the capability of creating routes and by dynamically preventing devices from accepting new children. 
During the tests, all packets sent by EDs were addressed to the network coordinator and included a payload of 77 bytes, corresponding to 50 ECG samples (12 bits each) and 2 control bytes. Each test run was executed at least twice and 1,000 messages were sent by each ED to the network coordinator. All messages were acknowledged hop by hop and used the KVP format. Up to 3 retransmissions were allowed. All experimental tests were carried out using channel 26, where the spectrum was relatively free of interference from Wi-Fi networks. The nodes were positioned relatively close to each other to avoid hidden nodes.

Fig. 2 (a) shows the smallest DR measured using sensors that transmitted raw ECG data and, consequently, tried to transmit a packet every $250 \mathrm{~ms}$. Fig. 2 (b) shows the smallest DR measured using data compression what reduces by half the amount of data transmitted by sensors, sending a packet every $500 \mathrm{~ms}$. As shown, both star networks were able to successfully relay all traffic generated by the EDs. However, as expected, the traffic increase in tree networks impacts their performance. In fact, it can be observed that deeper networks perform poorer than the ones with a small number of hops. It can be noticed that the 4-hop tree networks presents the worst performance, followed by the 3-hop tree network and so on. It can also be seen that tree networks that carry compressed traffic behave much better due to the lower traffic load.

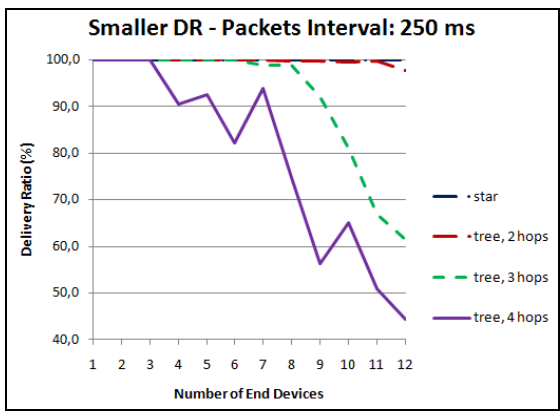

(a)

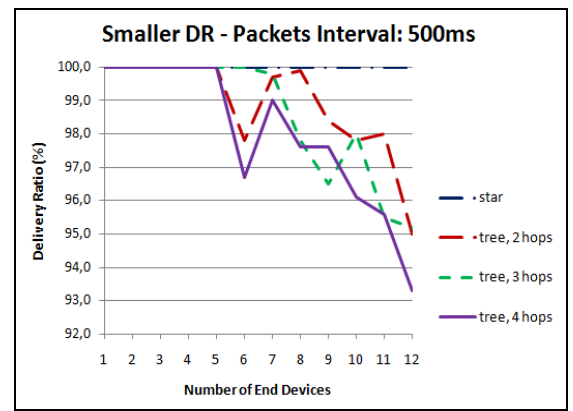

(b)

Fig. 2. Measured DR curves for a packet interval equal to $250 \mathrm{~ms}$ (a) and $500 \mathrm{~ms}$ (b).

Fig. 3 (a) and Fig. 3 (b) show the mean end-to-end delay curves when the interval between packets is equal to, respectively, $250 \mathrm{~ms}$ and $500 \mathrm{~ms}$. It can be observed that the mean delay experienced by packets in star, 2-hop and 3-hop tree networks is almost constant despite of the number of EDs. The same situation is not verified in 4- hop tree networks where the delay increases as a function of the number of EDs.

Fig. 4 (a) and Fig. 4 (b) show the maximum delay observed considering raw and compressed ECG data respectively. In the first case, the maximum delay observed for a 4-hop tree network was $360 \mathrm{~ms}$. If we consider that data is transmitted every 250 $\mathrm{ms}$, the total delay experienced by the first data sample collected is equal to $610 \mathrm{~ms}$ $(360 \mathrm{~ms}+250 \mathrm{~ms})$. The maximum delay observed when packets are transmitted every $500 \mathrm{~ms}$ was $181 \mathrm{~ms}$. In this case, the maximum delay experienced by a sample was equal to $681 \mathrm{~ms}(181 \mathrm{~ms}+500 \mathrm{~ms})$. These delay values are under acceptable limits 
for real-time waveform transmission out of intensive care units and operating rooms, according to the IEEE Std. 11073-00101-2008 [2].

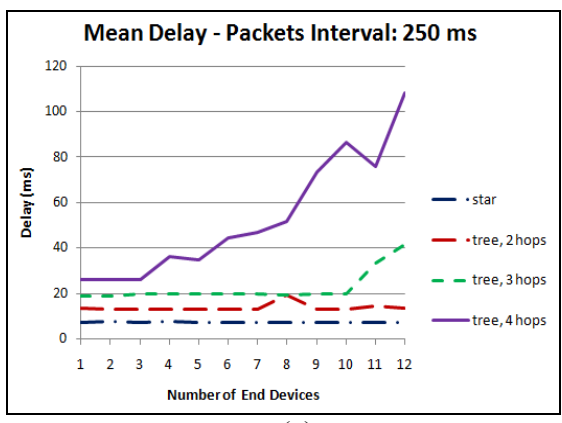

(a)

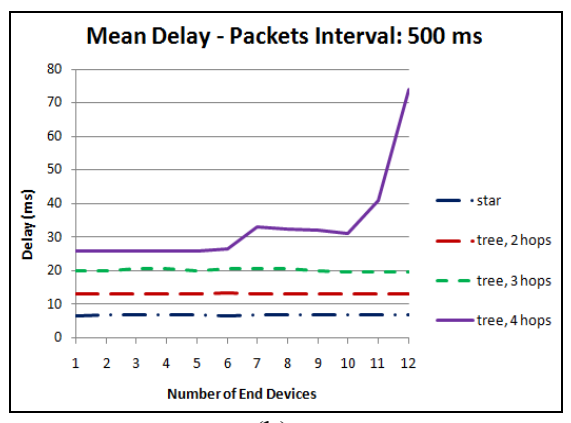

(b)

Fig. 3. Mean delay curves for the packet interval equal to $250 \mathrm{~ms}$ (a) and $500 \mathrm{~ms} \mathrm{(b).}$

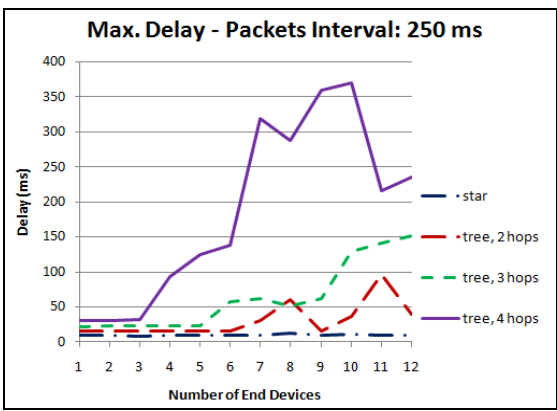

(a)

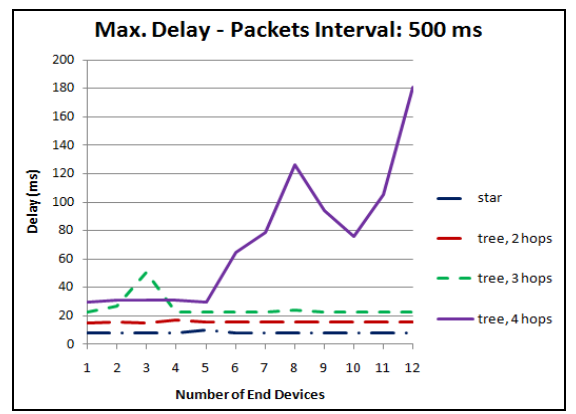

(b)

Fig. 4. Maximum delay curves for the packet interval equal to $250 \mathrm{~ms}$ (a) and $500 \mathrm{~ms}$ (b).

It is important to observe that some of the DR and delay curves presented are not monotonic, which means that, against to our expectations, the DR and delay values do not strictly decrease or increase as the number of end devices increases. It happens because test outcomes are highly dependent on the initial conditions, specifically on the time intervals between successive transmission attempts made by sensors. These intervals are initially established when sensors join the network and are, with small variations, maintained during the network operation. Nevertheless, we consider that the results obtained are representative and can be used when considering the design of a health monitoring systems based on the ZigBee protocol.

Table 1 summarizes our results. Both star networks were capable of achieving $100 \%$ DR. Even networks with several hops were capable of consistently relaying the traffic of a significant number of ECG sensors what could satisfy the requirements of health monitoring systems tailored to hospital scenarios such as emergency or ambulatory care. 
Table 1. Results summary.

\begin{tabular}{c|c|c|c|c|c|c}
\hline \multirow{2}{*}{$\begin{array}{c}\text { Network } \\
\text { topology }\end{array}$} & \multicolumn{2}{|c|}{$\begin{array}{c}\text { Max. number of EDs for } \\
D R=100 \%\end{array}$} & $\begin{array}{c}\text { Mean delay limit value } \\
(\mathrm{ms})\end{array}$ & \multicolumn{2}{c}{ Max. delay (ms) } \\
\cline { 2 - 7 } & $\Delta=250 \mathrm{~ms}$ & $\Delta=500 \mathrm{~ms}$ & $\Delta=250 \mathrm{~ms}$ & $\Delta=500 \mathrm{~ms}$ & $\Delta=250 \mathrm{~ms}$ & $\Delta=500 \mathrm{~ms}$ \\
\hline Star & $\geq 12$ & $\geq 12$ & 7 & 7 & 12 & 10 \\
Tree, 2 hops & 6 & 6 & 14 & 13 & 96 & 17 \\
Tree, 3 hops & 5 & 5 & 49 & 21 & 151 & 51 \\
Tree, 4 hops & 3 & 5 & 104 & 73 & 360 & 181 \\
\hline
\end{tabular}

Fig. 5. (a) DR (b) Mean Delay.

\section{Conclusions and future work}

In this work, the performance of ZigBee-based WSN for continuous patient monitoring is evaluated experimentally in terms of scalability considering different topologies. Only ECG nodes were considered because they are the most demanding in terms of traffic generated and are common in hospitals. Up to 12 ECG sensor nodes were employed and two operating modes were considered: raw ECG data transmission and compressed ECG data transmission. The test conditions were chosen to avoid two of the most common causes of packet loss and delay: the presence of interference and the occurrence of hidden nodes. Consequently, the outcomes are near optimum and can be used as references in the design of monitoring systems based on the ZigBee protocol.

Test results indicate that star networks operating in the unslotted CSMA-CA mode can relay all the traffic generated by at least 12 ECG nodes on both modes. In tree topologies, the increase of the network traffic load imposes a reduction in the number of devices, but they still can reliably relay the traffic of a considerable number of ECG nodes. Additionally, it was observed that packets do not experience large delays even in deep networks.

Future work includes testing several topologies in a real hospital environment, where several sources of interference and fading are present.

Acknowledgments. This work has been supported by the Portuguese Foundation for Science and Technology and the POCTI and FEDER programs.

\section{References}

1. ZigBee Alliance, ZigBee Health Care Profile Specification, Revision 15, Version 1.0. 2010: San Ramon, CA, USA.

2. IEEE, Health Informatics - PoC Medical Device Communication - Part 00101: Guide-Guidelines for the Use of RF Wireless Technology. IEEE Std 11073-00101-2008: p. 1-99.

3. Ilyas, M. and I. Mahgoub, eds. Handbook of Sensor Networks: Compact Wireless and Wired Sensing Systems. 1 ed., ed. C. Press. Vol. 1. 2004, CRC Press. 864. 
4. Fernandez-Lopez, H., et al., Evaluation of the Impact of the Topology and Hidden Nodes in the Performance of a ZigBee Network, in S-Cube 2009, S. Hailes, S. Sicari, and G. Roussos, Editors. 2009, ICST: Pisa, Italy. p. 256-271.

5. Fernández-López, H., et al., HM4All: A Vital Signs Monitoring System based in Spatially Distributed ZigBee Networks, in Second International Conference On Pervasive Computing Technologies For Healthcare, 2010. PervasiveHealth 2010. . 2010, IEEE (in press): Munich, Germany.

6. Tia, G., et al. Wireless Medical Sensor Networks in Emergency Response: Implementation and Pilot Results. in Technologies for Homeland Security, 2008 IEEE Conference on. 2008.

7. Patel, S., et al. Analysis of Feature Space for Monitoring Persons with Parkinson's Disease With Application to a Wireless Wearable Sensor System. in Engineering in Medicine and Biology Society, 2007. EMBS 2007.

8. Lorincz, K., et al., Sensor networks for emergency response: challenges and opportunities. Pervasive Computing, IEEE, 2004. 3(4): p. 16-23.

9. Yuanlong, L. and R. Sahandi. Zigbee network for remote patient monitoring on general hospital wards. in Information, Communication and Automation Technologies, 2009.

10. Ericsson. Ericsson Mobile Health. [cited October, 2007]; Available from: http://www.ericsson.com/solutions/enterprise/products/mhealth solutions.shtml.

11. Intelesens. Intelesens Sensors. 2010 [cited 2010 March 2nd, 2010]; Available from: http://www.intelesens.com/sensors/index.html.

12. Latre, B., et al., Throughput and Delay Analysis of Unslotted IEEE 802.15.4. Journal of Networks, 2006. 1(1): p. 20-28.

13. Liang, X. and I. Balasingham, Performance Analysis of the IEEE 802.15.4 based ECG Monitoring Network, in Proceedings of the 7th IASTED International Conferences on Wireless and Optical Communications. 2007: Montreal, Canada.

14. Golmie, N., D. Cypher, and O. Rebala, Performance Analysis of Low Rate Wireless Technologies for Medical Applications. Computer Communications, 2005. 28: p. 12661275.

15. Casas, R., et al., ZigBee-based alarm system for pervasive healthcare in rural areas. Communications, IET, 2008. 2(2): p. 208-214.

16. IEEE, IEEE Std 802.15.4-2003-Part 15.4: Wireless LAN Medium Access Control (MAC) and Physical Layer (PHY) Specifications for Low-Rate Wireless Personal Area Networks (LR-WPANS), IEEE, Editor. 2003.

17. ZigBee.Alliance, ZigBee Alliance Document 053474r17, ZigBee Specification, v. 1.0 r17, Z. Alliance, Editor. 2007.

18. Jennic. Product Brief - JN5139-xxx-Myy IEEE802.15.4/ZigBee Module Family. 2008 [cited May, 2008]; Available from: http://www.jennic.com/products/index.php?productID $=0000000002$. 